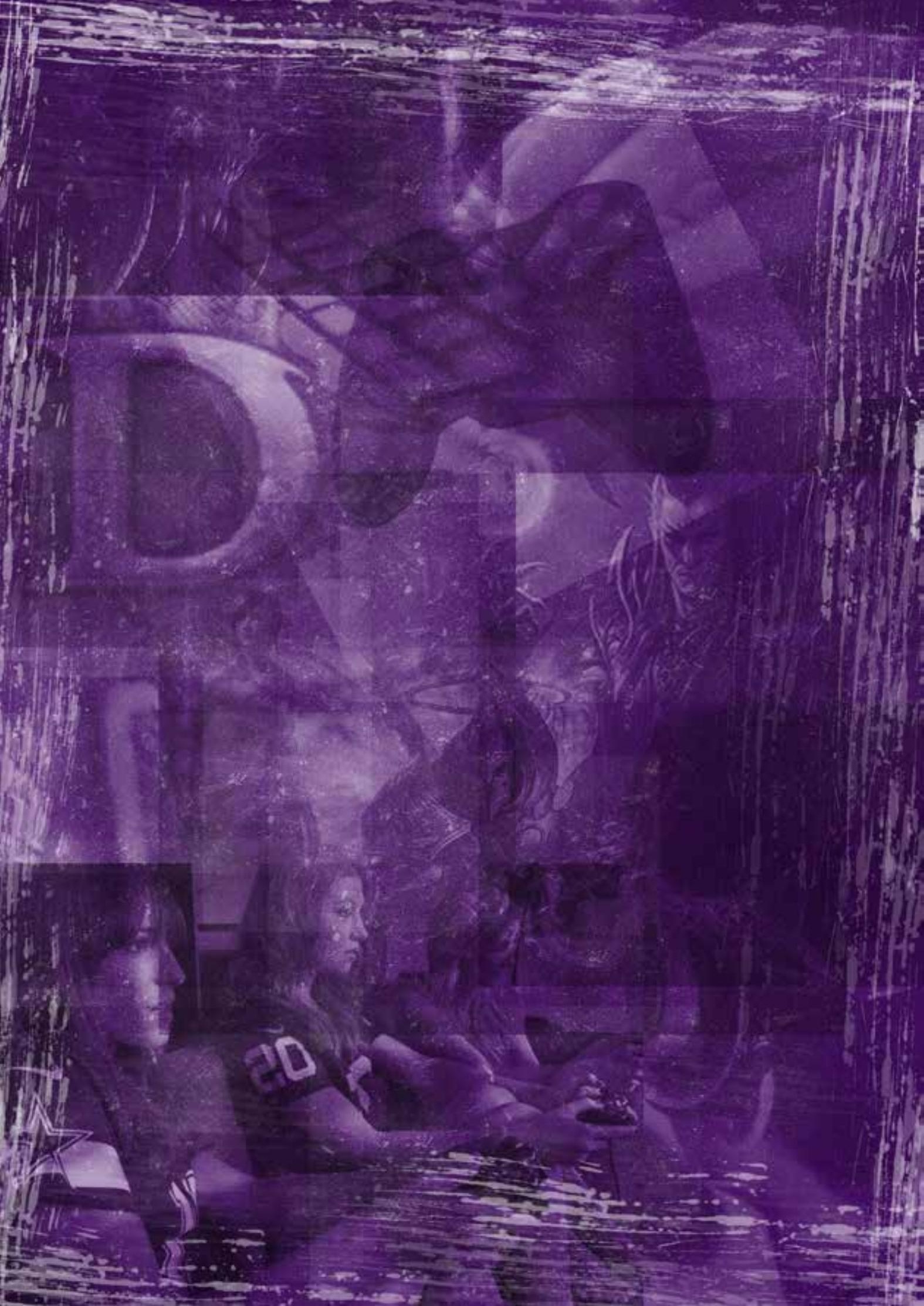




\section{Coreografías de género en espacios de juego online. Jugadoras, fans y conflictos en videojuegos}

Gender choreographies in online game spaces. Female players, fans and gender conflicts in video games.

DoI: $10.15213 /$ redes.n11.p154

HÉCTOR PUENTE BIENVENIDO Y AMPARO LASÉN DÍAZ

\section{ABSTRACT}

This analysis of the players and fans of Diablo III (2012) discusses gender choreographies as forms of shared agencies (LATOUR, 1994; LASÉN, 2012) among players and features of the game with the background of sexism and stereotypes, which may be actualized, ignored or combated in practices (TAYLOR, 2006; CORNELIUSSEN AND RETTBERG, 2008); considering that game practices may contribute to construct gender and destabilize certain existing expectations.

KEYWORDS: ENDER, VIDEO GAMES, AGENCY, MEDIA STUDIES, FAN CULTURE.

\section{RESUMEN}

En este artículo proponemos un análisis de los y las jugadoras y fans de Diablo III (2012), poniendo el acento en las coreografías de género que se desarrollan como formas de agencia compartida (LATOUR, 1994; LASÉN, 2012) entre jugadores, jugadoras y características del juego con el trasfondo del sexismo y los estereotipos, que pueden ser actualizados, ignorados o combatidos en las prácticas (TAYLOR, 2006; CORNELIUSSEN Y RETTBERG, 2008); viendo cómo las prácticas de juego pueden contribuir a hacer género y a desestabilizar ciertas expectativas existentes.

PALABRAS CLAVE: VIDEOJUEGOS, AGENCIA, media studies, CULTURA FAN 


\section{INTRODUCCIÓN Y OBJETO}

Inspirados por los trabajos pioneros de Justine Cassel y Henry Jenkins (2000) en el campo del género y los videojuegos, numerosos autores han abordado las problemáticas relativas a las mujeres en el mundo del software lúdico. Desde el ámbito de estudios literarios, comunicativos y narratológicos (KENNEDY, 2002; LAUREL, 2006,), sociológicos y culturales (TAYLOR, 2006; PEARCE, 2009; SARKEESIAN, 2013), metodológicos (HEETER, 2008) o de corte multidiciplinar $^{1}$ (KAFAI ET AL., 2008; EGENFELDT-NIELSEN, SMITH Y TOSCA, 2012) las videojugadoras han suscitado recientemente un alto grado de interés entre los académicos y estudiosos del juego, principalmente motivado por la tradicional carencia de información e infrarrepresentación en la industria. Representaciones, estereotipos, prácticas, o fenómenos más controvertidos como el sexismo, han recibido una especial atención. Concretamente, este último aspecto ya había sido abordado por Schott y Horrel (2000), quienes en un extenso trabajo, a partir de entrevistas personales con videojugadoras, concluyen que las mujeres reclaman una representación de género más equilibrada y realista, reivindicando una mayor variedad de estudios de caso.

Pese a todo, si bien parece existir un fuerte consenso en que las cuestiones relativas a las videojugadoras de rol aún no han sido suficientemente abordadas (CASSEL Y JENKINS, 2000; PEARCE, 2009), en el caso español apenas disponemos de estudios rigurosos específicamente centrados en las mujeres y el gaming. Tal y como afirma Taylor (2008), los estudios de caso en profundidad, además de servir para iluminar procesos sociales más amplios, tienen un gran potencial como reveladores de las prácticas y significados de las culturas locales y participantes.

Indagar en las coreografías de género (FOSTER, 1998) que se dan en las prácticas de juego online puede visibilizar y hacernos comprender mejor los mecanismos subyacentes que operan en las agencias e interacciones, revelando cómo el juego construye género y prácticas desiguales (limitaciones, resistencias...). De este modo, ante las advertencias y reclamos de mayor precaución, sensibilidad o pluralidad de estudios de caso, Ermi et al. (2005), Mäyrä (2009) o Puente y Fernández (2013) consideran que es muy importante tener en cuenta la diversidad cultural y los contextos específicos donde el juego toma lugar y es practicado; por ello, adoptar una postura sensible a las cuestiones de género se vuelve indispensable.

1 Enténdiendose por multidisciplinariedad en el ámbito de los games studies (narratología, ludología, diseño, situacionismo, estudios socio-culturales ...). 
A modo introductorio, el trabajo que aquí se presenta, aspira a contribuir y aumentar la necesaria pluralidad reclamada, aportando nuevos matices, miradas y entendimientos respecto a las prácticas, agencias compartidas y coreografías de género que se desarrollan en los espacios lúdicos.

En este artículo presentamos un análisis que nace de la investigación realizada durante cuatro años de proyecto de tesis ${ }^{2}$, en la que se recopilaron ingentes cantidades de información a partir de una etnografía virtual, entrevistas en profundidad y encuesta online a 956 jugadores de Diablo III $^{3}$. En este artículo proponemos un estudio de los y las jugadoras y/o fans de Diablo III 4(2012), poniendo el acento en las coreografías de género que se desarrollan como formas de agencia compartida (LATOUR, 1994; LASÉN, 2012) entre jugadores, jugadoras y características del juego con el trasfondo del sexismo y los estereotipos, que pueden ser actualizados, ignorados o combatidos en las prácticas (TAYLOR, 2006; CORNELIUSSEN Y RETTBERG, 2008); viendo cómo las prácticas de juego pueden contribuir a hacer género y a desestabilizar ciertas expectativas existentes.

Comenzaremos abordando las nociones de agencia compartida y las coreografías de género para posteriormente exponer la metodología y realizar un análisis detallado acerca de los hábitos y consumo de juegos, por parte de jugadoras y fans, bajo el trasfondo del sexismo. La última sección trata del consumo, práctica y representación de los personajes femeninos, mientras proponemos un acercamiento a los espacios de juego desde la óptica de los conflictos que se presentan en las coreografías de género.

\section{AGENCIA COMPARTIDA Y COREOGRAFÍAS DE GÉNERO}

En este trabajo entendemos las prácticas de juego online como una forma de agencia compartida entre las jugadoras y los jugadores, los dispositivos

2 Tesis de Héctor Puente Bienvenido: Interacción, performatividad y sociabilidad en espacios de juego en red. El caso de los jugadores españoles de género rol(Diablo III).

3 Diablo III es un célebre videojuego de rol de acción multijugador (aRPG) con récord histórico de ventas en su día de lanzamiento (con más de 3,5 millones de copias vendidas en menos de 24 horas) En la actualidad cuenta con más de 20 millones de unidades vendidas. Disponible en: http://eu.battle. net/d3/es/blog/10033561/diablo-iii-r\%C3\%A9cord-hist\%C3\%B3rico-en-ventas-para-pc-el-d\%C3\%ADadel-lanzamiento-23-05-2012 
digitales, las distintas plataformas y sus condiciones de diseño y comercialización. Al hablar de agencia compartida entendemos que la capacidad de hacer, decir, representar y actuar de jugadores y jugadoras depende en parte de los rasgos, potencialidades y limitaciones de los dispositivos tecnológicos, tanto en sus aspectos técnicos como respecto de las condiciones de comercialización marcadas por operadores, servidores y propietarios de las plataformas. A su vez, dichos rasgos no determinan totalmente los usos, ya que los usuarios y usuarias movidos por sus deseos, intenciones, ideas y prejuicios, y también por la obligaciones y expectativas que se generan en las relaciones mediadas por la práctica de juego, actualizan o desestiman las posibilidades técnicas de actuación, al tiempo que pueden favorecer la emergencia de ciertos usos que habían sido diseñados por los creadores y comercializadores de los juegos. De tal manera que podemos decir que los dispositivos son producidos también por los usos, que en las prácticas de juego se siguen diseñando los juegos.

Esta agencia compartida no comprende solamente al individuo y a los dispositivos digitales, sino que se trata de una suerte de coreografía colectiva, de la que participan jugadores, diseñadores, comercializadores, en toda su heterogeneidad y con intereses contrapuestos, de manera que no puede entenderse a priori sólo como una forma de colaboración, sino que también revelan conflictos y constreñimientos, una dinámica hecha de encuentros y de encontronazos, un baile no exento de controversias y pisotones. Así nos encontramos con una configuración cultural, social y personal de las tecnologías, pero también, recíprocamente, las personas, sus cuerpos y sus relaciones se ven transfiguradas por los usos y mediaciones tecnológicos, a través de esta agencia compartida entre personas y tecnologías, donde no sólo están implicados usuarios y artefactos, sino también las condiciones de comercialización marcadas por operadores, servidores y propietarios de las plataformas, y las distintas regulaciones institucionales e informales de dichos usos y prácticas, esto es, las normas y regulaciones de uso, tanto las formales e institucionalizadas, como las convenciones y las reglas no escritas de etiqueta, de lo que es apropiado y lo que no. Todos estos elementos forman procesos de uso y aprendizaje que cambian en el tiempo.

Esas relaciones que conforman estas formas de agencia compartida son también relaciones de género, donde se hace género y a veces son cuestionadas las expectativas y atribuciones de género dominantes. De ahí que las prácticas de juego sean actividades donde en la actualidad se configuran también coreografías de género (FOSTER, 1998). El sociólogo estadounidense Erwing Goffman es el primero en proponer esta visión coreográfica del género como dispositivo 
de organización social: "Who is brought or brings himself into the immediate orbit of another; who initiates talk, who is selected as the addressed recipient, who self-selects in talk turn-taking, who establishes and changes topics, whose statements are given attention and weight, and so forth" (GOFFMAN, 1977: 324), quién decide, quién lleva y quién sigue en el baile de las interacciones, cara a cara o mediadas digitalmente, dónde otros órdenes y jerarquías se actúan también (profesional/doméstico, público/privado).

Entender las relaciones de género como coreografías, subraya además del carácter relacional del género la reciprocidad de los posicionamientos, cómo las posiciones de unos determinan o limitan los movimientos de las otras y cómo salirse de esos movimientos conlleva el riesgo de pisarse o empujarse. Hablar de coreografía de género en lugar de performance o actuación de género permite mantener las consideraciones perfomativas del género (BUTLER, 1990), esto es, la consideración del género como formas de actuación repetidas, que actualizan normas, encarnadas por las personas en sus relaciones e interacciones, subrayando también la importancia del script, del guión, del ordenamiento que se actualiza, se repite, "se baila". También la noción de coreografía nos recuerda la pluralidad de sus modalidades: junto a los bailes de salón asimétricos (el que abraza y es abrazado, el que lleva y es llevado), existen otras formas de danza, como aquellas en las que todos se mueven al unísono, a la imagen de las masas rítmicas descritas por Elias Canetti, como las coreografías de la música disco o las de los videos pop de Beyoncé o Lady Gaga recreadas por tantos de sus fans; y también otras en las que los bailarines juegan con la simetría y la diferencia, los contactos y las resonancias, sin que estén fijas las posiciones de la que lleva y es llevado, o donde ni siquiera esté claro que alguien lleve a alguien.

Las coreografías de género contemporáneas están con mucha frecuencia mediadas y remediadas digitalmente, en el sentido de remediación tal y como lo describen Bolter y Grusin (2000), esto es, sujetas a una mediación extendida y múltiple, tanto en cuanto a los medios y tecnologías implicados como a las esferas de la vida diaria y relaciones donde se dan estas mediaciones; a la vez que uno de los motores de esa mediación extensa y extendida es un ideal de inmediatez y transparencia. De forma que esas coreografías son el resultado de una agencia compartida entre personas y medios, en este caso entre jugadores, jugadoras y todos los dispositivos y software implicados en los videojuegos online, y en nuestro caso los rasgos particulares del juego Diablo III. Esta agencia compartida contribuye a establecer las normas contextuales que definen lo que es apropiado y las expectativas mutuas dentro del juego. Observar el caso específico de cómo se movilizan las normas y expectativas 
acerca de las interacciones dentro del juego en relación con el género permite ver cómo las coreografías de género refuerzan o minimizan diferentes características de los juegos online, así como ver de qué manera las situaciones de juego cuestionan o refuerzan los estereotipos de género, lo que se considera femenino y masculino.

\section{METOdOLOGÍA DE INVESTIGACIÓN}

Para la realización del trabajo y discusión propuesta se ha optado por rescatar algunos de los datos obtenidos durante el trabajo de campo sometiéndolos a un nuevo tratamiento más orientado y sensible a las relaciones, coreografías y estereotipos de género que se manifiestan en los videojuegos de rol online (así como a todas las prácticas de resistencia, pisotones o reconfiguración en torno a estos). Bajo un diseño metodológico mixto, más conocido como triangulación de métodos (DENZIN, 1970), combinamos técnicas cuantitativas y cualitativas de investigación que se concretan en la observación participante en red (más de dos años de trabajo etnográfico de campo), entrevistas en profundidad y datos procedentes de encuestas online $e^{5}$ a 956 jugadores y jugadoras de Diablo III seleccionados a través de un muestreo intencional ${ }^{6}$ (y posteriormente sometidos a técnicas de análisis multivariante).

\section{MOdOS DE ACCESO Y PRÁCticas de LAS FANS Y JUGAdoras EN DIABLO III}

Acceder a la realidad de las mujeres y sus prácticas en los juegos de rol online no siempre resulta del todo sencillo. Un número reducido de jugadoras (CORNELIUSSEN Y RETTBERG, 2008), junto a una densa carga de prejuicios, roles adscritos e inercias (BECKER, 1995), distorsionan y dificultan grandemente la compresión de la presencia de las jugadoras en los juegos de rol online. Especialmente relevantes para el entendimiento de los videojuegos y prácticas lúdicas (TURKLE 7 1995), pero tradicionalmente invisibilizadas,

5 El trabajo de campo de la encuesta online se realizó durante el mes de mayo de 2013 (período en el que la encuesta estuvo activa). El diseño del cuestionario se puede consultar en el siguiente enlace: https://es.surveymonkey.com/s/encuesta_diablo_hpb

6 En el muestro intencional, típico de diseños cualitativos, la selección de las unidades muestrales se realiza a partir de la experiencia, criterio o juicio de los investigadores.

7 Sherry Turkle es una de las iniciadoras de los game studies. Sus estudios iniciales se desarrollaron en MUDs (Multi user domain) y se centraron en aspectos relacionados con el modo en el que los 
infrarrepresentadas o incluso obviadas (CASSEL Y JENKINS, 2000; PEARCE, 2009; TAYLOR, 2006) las videojugadoras, como agente fundamental en las prácticas de juego, merecen una especial atención.

En este apartado trataremos primero del acceso, presencia y articulación de problemáticas relativas a las jugadoras en Diablo III, describiendo cuál es la situación actual de las usuarias y fans en el servidor, para posteriormente analizar algunas de las desavenencias y desasosiegos surgidos cuando las actividades y coreografías de género entran en conflicto con los discursos hegemónicos y las prácticas adscritas a rol (GOFFMAN, 1959), prestando especial atención al trasfondo de los estereotipos y el sexismo.

Los modos en los que las mujeres se inician en los videojuegos de rol de acción, y más concretamente Diablo III, son sustancialmente diversos y heterogéneos. Desde fórmulas y vías más tradicionales y/o cotidianas (experiencias previas, recomendaciones, mera curiosidad o interés) hasta cauces de acceso mucho menos usuales $\mathrm{u}$ ordinarios (tales como accesos casuales accidentales). Según Taylor (2006), pese a que muchos usuarios empiezan a jugar gracias a familiares o amigos, serían las videojugadoras (junto a los power gamers) quienes tienden especialmente a acceder mayoritariamente por este tipo de vías a través de un contacto cercano8.

FiguRA. 1. GRÁFICO DE DATOS REFERENTE A LAS VÍAS DE INTRODUCCIÓN A LOS VIDEOJUEGOS.

jugadores interactuaban en el juego y construían su identidad.

8 Se entiende por contacto cercano algún miembro de grupo de socialización primario o secundario. 


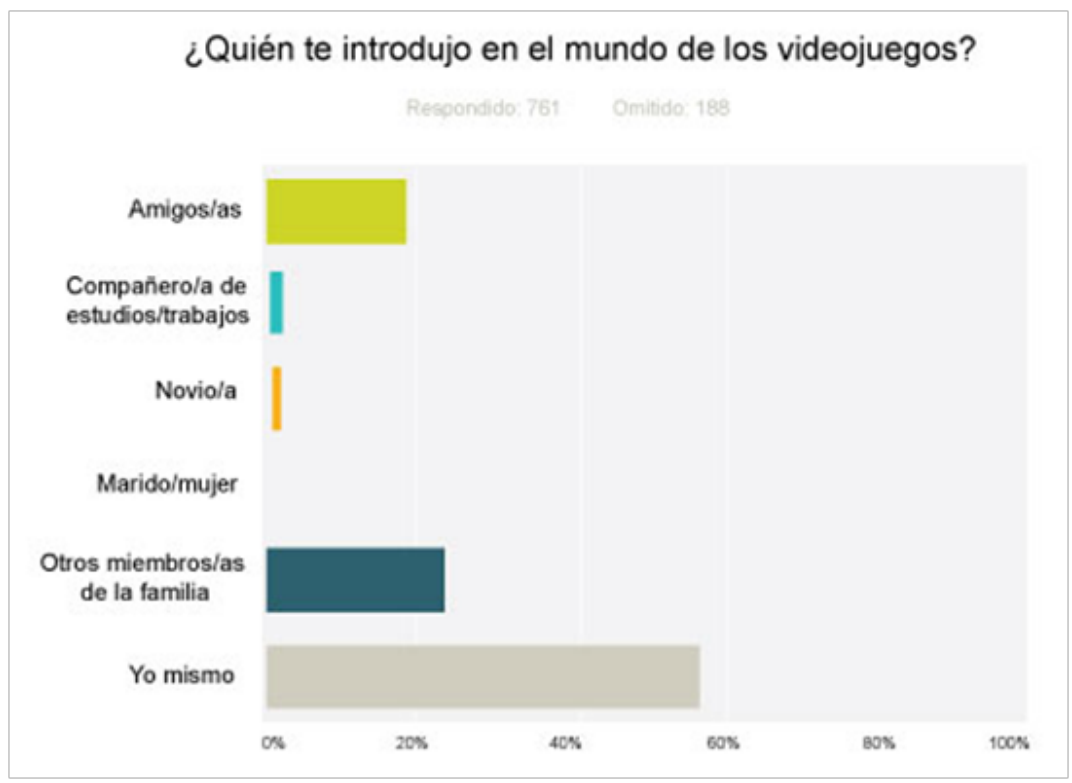

\begin{tabular}{l|cc}
\multicolumn{1}{c|}{ OPCIONES DE RESPUESTA } & \multicolumn{2}{c|}{ RESPUESTAS } \\
\hline Amigos/as & $17,74 \%$ & 135 \\
\hline $\begin{array}{l}\text { Compañeros/a de estudios/ } \\
\text { trabajo }\end{array}$ & $1,58 \%$ & 12 \\
\hline Novio/a & $1,31 \%$ & 12 \\
\hline Marido/Mujer & $0,26 \%$ & 2 \\
\hline $\begin{array}{l}\text { Otros miembros/as de } \\
\text { la familia (distintos a los } \\
\text { mencionados) }\end{array}$ & $23,00 \%$ & 175 \\
\hline Yo mismo & $56.11 \%$ & 427 \\
\hline Total & & 761 \\
\hline
\end{tabular}

Fuente: Creación propia A partiR de Datos de ENCUESTA ONLINE

El gráfico 1 ilustra los diferentes modos de acceso a la práctica del videojuego. Frente a una leve mayoría $(56,11 \%)$ que afirma haberse iniciado por sí misma, el total restante $(43,89 \%)$ reconoce que accedió por primera vez a través de algún familiar, amigo y/o conocido (véanse categorías de respuesta 
adicionales). Pero si las mujeres ${ }^{9}$ tienden a acceder al software lúdico por primera vez a través de contactos cercanos, ¿Ocurre esto mismo en el caso de las jugadoras de Diablo III? Para responder a esta cuestión en el siguiente gráfico se cruza la variable sexo con la información relativa al primer contacto con el mundo de los videojuegos. A través de una prueba de chi-cuadrado se contrasta si existe algún tipo de asociación entre las variables sexo y la vía de introducción al videojuego. Los datos obtenidos revelan una asociación estadísticamente significativa entre las variables mencionadas, tal y como se detalla en los gráficos que se muestran a continuación.

Figura. 2. TABla de CONTINGENCIA (CRUCE ENTRE SEXo y PRIMER CONTACTO CON VIDEOJUEGOS)

\section{Tabla de contingencia Sexo * ¿Quién te introdujo en el mundo de los videojuegos?}

\begin{tabular}{|c|c|c|c|c|c|c|c|}
\hline Sexo & $\begin{array}{l}\text { Amigos/ } \\
\text { as }\end{array}$ & $\begin{array}{l}\text { Compañeros } \\
\text { de estudio/ } \\
\text { trabajo }\end{array}$ & Novio/a & $\begin{array}{l}\text { Marido/ } \\
\text { Mujer }\end{array}$ & $\begin{array}{l}\text { Otros } \\
\text { miembros } \\
\text { de la familia } \\
\text { (distintos } \\
\text { a los } \\
\text { mencionados) }\end{array}$ & $\begin{array}{l}\text { Yo } \\
\text { mismo }\end{array}$ & Total \\
\hline Hombre & 130 & 12 & 2 & 1 & 169 & 410 & 724 \\
\hline Mujer & 4 & 0 & 7 & 1 & 6 & 17 & 35 \\
\hline Total & 134 & 12 & 9 & 2 & 175 & 427 & 759 \\
\hline
\end{tabular}

9 Los datos disponibles en los games studies referentes a las videojugadoras provienen de fuentes principalmente norteamericanas y escandinavas. Existe una gran carencia de estudios de caso en el ámbito nacional español, por lo que la profundización en análisis, tanto cualitativos como cuantitativos, focalizados en videojugadores españoles pueden arrojar mucha luz sobre numerosos interrogantes en el campo de estudio mencionado. 


\section{Pruebas de chi-cuadrado}

\begin{tabular}{|l|c|c|c|}
\hline & VALOR & GL & $\begin{array}{c}\text { SIG. ASINTótica } \\
\text { (BILATERAL) }\end{array}$ \\
\hline $\begin{array}{l}\text { Chi-cuadrado de } \\
\text { Pearson }\end{array}$ & 121,227 & 5 &, 000 \\
\hline $\begin{array}{l}\text { Razón de verosimi- } \\
\text { litudes }\end{array}$ & 40,264 & 5 &, 000 \\
\hline $\begin{array}{l}\text { Asociación lineal por } \\
\text { lineal }\end{array}$ &, 346 & 1 &, 556 \\
\hline N de casos válidos & 759 & & \\
\hline
\end{tabular}

Fuente: creación propia a partir de datos de encuesta online.

A partir de la prueba de chi-cuadrado ${ }^{10}(\mathrm{p}$-valor $<0,05)$, rechazamos la hipótesis nula (Ho) de independencia entre variables. De este modo, hallándose diferencias estadísticamente significativas, aceptamos la hipótesis alternativa (Hi) de que existe algún tipo de asociación entre el sexo (variable independiente) y la vía de acceso o introducción a los videojuegos (variable dependiente). Se confirma, por tanto, que las jugadoras de Diablo tienen una mayor tendencia que los hombres a acceder a los videojuegos a través de familiares cercanos ${ }^{11}$ y/o amigos. A modo de ejemplo, de especial interés resultó la categoría de respuesta novio/ $\mathrm{a}^{12}$, donde una quinta parte de las jugadoras respondientes ( 7 sobre 35) afirmaron haber accedido al mundo del software de entretenimiento a través de su pareja ${ }^{13}$; frente a tan sólo un $0,15 \%$ de los varones ( 2 sobre 724$)^{14}$. En el caso de acceso por vía individual el porcentaje de hombres supera en

10 Cuando el p-valor resultante (dato marcado en amarillo en la ilustración) es menor a 0,05 rechazamos la hipótesis nula de independencia entre variables (en este caso sexo y vía de acceso a los videojuegos).

11 Considerándose a familiares cercanos (novio/a, marido/mujer y otros miembros de la familia).

12 Dicha categoría de respuesta fue incluida, en la encuesta online, gracias a las recomendaciones de los revisores del pre-test. Ejemplos como éste, demuestran la gran importancia de testear la encuesta online antes de su lanzamiento definitivo al campo.

13 Dado que no conocemos el sexo de la pareja, tan sólo del respondiente, no es posible inferenciar el sexo del novio/a, por lo que sería un error asumir que todos los entrevistados sean heterosexuales.

14 La misma tendencia encontrada en la categoría novio/a se mantiene en el caso de la categoría marido/mujer. 
nueve puntos a las mujeres ya que un 57\% de los hombres afirma haberse introducido por sí solo a los videojuegos, frente a un $48 \%$ de las mujeres usuarias.

En el trabajo etnográfico se encontraron numerosos ejemplos de jugadores que regalan a sus novias distintas plataformas y dispositivos de ocio interactivo con el objetivo de introducirlas en el mundo del software lúdico; o con la finalidad de disponer de una plataforma de juego en el domicilio de sus parejas para uso o beneficio propio.

"Sí, alguna vez, de hecho [a mi novia] la regalé una consola, la Wii y la he descargado algún juego para el ordenador, como los Sims y eso...”.

(ENTREVISTA No: 1. HOMBRE, 31 AÑOS, MADRID).

En una línea similar se observan distintos ejemplos de jugadoras que accedieron a Diablo por primera vez a través de recomendaciones, sugerencias o incluso leves y sutiles presiones por parte de sus respectivas parejas. Mientras, la tendencia contraria, varones introducidos por sus novias/os, es mucho menos habitual. Por lo que las coreografías de género en los espacios de juego en este aspecto corresponderían a un baile asimétrico donde los hombres inician y llevan a las mujeres.

"Diablo me lo recomendó un amigo, porque claro son muchos años y los fans de Diablo esperando a que saliese Diablo III... "venga cómpratelo que vamos a jugar...tal", "Bueno, venga" estuve viendo unos días en qué consistía y tal, me gustó la idea, me lo compré...y la verdad es que me encantó, me pareció un mundo muy adictivo y muy divertido" (ENTREVISTA N ${ }^{\circ}$ : 9. MUJER, 23 AÑos, GETAFE).

"Y nada, la verdad es que yo no estaba interesada en Diablo para nada, o sea, que el género no me motivaba nada la verdad. Pero mi chico, que es un poco pesado, pues me fue introduciendo y tal... presionando poco a poco $y$ demás" (ENTREVISTA No: 7. MUJER, 20 AÑOS, TOLEDO).

"Porque a mi novio bueno claro encantado de la vida de que juegue a Diablo, porque así juego con él y eso..." (ENTREVISTA No: 5. MUJER, 27 AÑos, BARCELONA).

Un ejemplo particularmente interesante, e interesado, de cómo los varones inician a sus parejas en el juego es el singular caso de una serie de jugadores que utilizaban a sus novias como farmeadoras, esto es, empleándolas en 
el servidor como instrumentos para la consecución de recursos virtuales de diversa índole (dinero, ítems, experiencia...). Mientras las riquezas logradas engrosaban las cuentas virtuales y privadas de juego, los jugadores varones se dedicaban a otro tipo de prácticas y actividades en escenarios simultáneos, como jugar al célebre juego de fútbol online, FIFA. De este modo, algunos hombres introducen a sus novias en los espacios de juego con una finalidad puramente instrumental o utilitarista, empleando a sus respectivas parejas para avanzar lo más rápida y exitosamente posible en el transcurso del juego, siendo notablemente más competitivos en el servidor. Esta particular coreografía de género es un ejemplo claro de agencia compartida entre las intenciones de los jugadores y las disposiciones del juego.

"Y respecto a lo de cómo llegué a interesarme...pues te cuento. Mi novio juega muchas tardes y noches que tiene libre, ya sea al Diablo o a otros juegos, y hacen grupos y tal para jugar... Pues uno de sus amigos, que es muy, muy friki ponía a su novia a jugar para ganar experiencia, oro, objetos...lo que sea, es decir, lo que necesitaran en ese momento. Entonces cuando él estaba ocupado, o no estaba, pues ponía a la novia a jugar con su personaje. Pues, bueno...pues básicamente mi chico empezó a hacer lo mismo conmigo de una manera más sutil. Cuando se ponía a jugar al fifa con su compañero de piso, que tenían torneos y tal, pues me decía, "oye, ¿te importaría jugar un rato con mi bárbaro al Diablo? Que si no me quedo descolgado", y entonces me decía, "mira lo único que tienes que hacer es seguir al grupo e ir haciendo lo que ellos te digan, les sigues con el ratón y das a estos dos botones, y eso, lo que ellos te vayan diciendo". Y eso, así me introduje más o menos". (ENTREvista MUJER, 20 AÑOS, TOLEDO).

Las consecuencias de este contacto inicial con el juego fueron totalmente insólitas. La interacción con otras jugadoras en una situación similar empleadas como farmeadoras originaron una serie de rutinas y afectividades de juego que desembocaron en una toma de decisiones que podríamos llamar emancipadora. Las usuarias mencionadas, cansadas de la situación, decidieron comprarse una copia del juego para su propio uso, manifestando explícitamente que si tenían que farmear lo harían para sí mismas. Los vínculos afectivos surgidos a partir de las rutinas de juego motivaron, sustancialmente, la decisión de compra, en un ejemplo de cómo la experiencia con los dispositivos participa 
en nuestros procesos de subjetivación y cómo desde las interacciones sociales generamos orden, ordenamiento, así como cuestionamos atribuciones y expectativas de género. De este modo, se evidencian a la perfección algunos de los roces, traspiés o conflictos que surgen de esas danzas y coreografías de género, el baile que iniciaron estos varones al introducir a sus novias en el juego con el fin de que "trabajaran" para ellos, se transformó en una toma de decisión autónoma de las mujeres apoyadas en las experiencias de juego con otras jugadoras, así como en el propio placer encontrado en la práctica del videojuego.

"Así tuve mi primer contacto directo. Pero sinceramente, era un poco rollazo, porque era básicamente farmear y farmear sin más. Además, no te enteras muy bien de cuál es el cometido de las cosas. Pero un día la novia del amigo de mi chico, estábamos las dos farmeando y me dijo algo como "¿por qué no nos hacemos nosotras un personaje para nosotras?”. Y así por aburrimiento, rutina, o por lo que fuera, pues nos decidimos a hacernos unos personajes, ella su cazadora de demonios y yo mi maga. Y la verdad es que ahí es cuando te engancha, cuando empiezas de cero, porque el juego cambia radicalmente, o sea, la percepción del juego una vez que te sumerges en la historia, la trama, en el juego en sí, cambia, cambia muchísimo. $\mathrm{Y}$ ahí es cuando nos interesamos y cuando ya me decido a comprarme yo el juego para tener mi propia cuenta”. (ENTREVISTA MUJER, 20 AÑos, TOLEDO).

\section{HÁbITOS Y CONSUMO DE JUEGO CON EL TRASFONDO DEL SEXISMO}

Según las estadísticas recopiladas por Egenfeldt-Nielsen, Smith y Tosca (2012) las mujeres juegan menos horas y están menos comprometidas que los hombres, debido principalmente a lo que identifican como una escasez de contenido atractivo para las jugadoras. Sin embargo, nuestros hallazgos, relativos al género rol de acción parecen poner en cuestión dichas afirmaciones. El compromiso no puede ser medido únicamente por las horas de juego directo, pese a que en la literatura especializada y en los informes disponibles así se ha hecho hasta la fecha, ya que también se ha de tomar en cuenta la inversión de tiempo e interés fuera del espacio de juego en sí, por ejemplo, en la consulta o producción de contenido generado por el usuario inspirado en el universo de Diablo III. Pero es que además, en nuestro caso el tiempo medio invertido en el juego diariamente es muy similar entre hom- 
bres y mujeres, es más éste sería levemente superior entre las jugadoras de Diablo III (2,17 horas diarias frente a 2,03 horas de los varones).

También es cuestionable la creencia de que los hombres gastan más dinero en esta actividad que las mujeres. A la vista de una prueba de chi-cuadrado podemos concluir que no existirían diferencias estadísticamente significativas $^{15}$ entre jugadores y jugadoras de Diablo III en cuanto a gasto mensual en videojuegos (el gasto medio es levemente superior en el caso de los hombres). Sin embargo, tal y como se observa en las tablas que se ofrecen a continuación, el porcentaje de jugadores que no invierten ninguna cantidad al mes (o euros) es levemente superior entre la muestra de varones $(27,2 \%$ frente a $22,2 \%$ de las mujeres).

Figura. 3. Distribución DE FRECUENCIAS INVERSión MEdia MUjeRES (MENSUAL).

\section{¿Cuál es tu inversión mensual en videojuegos? MUJERES}

\begin{tabular}{|c|c|c|c|c|c|}
\hline & & FRECUENCIA & PORCENTAJE & $\begin{array}{l}\text { PORCENTAJE } \\
\text { VÁLIDO }\end{array}$ & $\begin{array}{l}\text { PORCENTAJE } \\
\text { ACUMULADO }\end{array}$ \\
\hline \multirow{7}{*}{ Válidos } & o euros & 8 & 22,2 & 23,5 & 23,5 \\
\hline & $\begin{array}{c}\text { de } 1 \text { a } 20 \\
\text { euros }\end{array}$ & 16 & 44,4 & 47,1 & 70,6 \\
\hline & $\begin{array}{c}\text { de } 21 \text { a } 40 \\
\text { euros }\end{array}$ & 6 & 16,7 & 17,6 & 88,2 \\
\hline & $\begin{array}{c}\text { de } 41 \text { a } 60 \\
\text { euros }\end{array}$ & 2 & 5,6 & 5,9 & 94,1 \\
\hline & $\begin{array}{c}\text { de } 61 \text { a } 80 \\
\text { euros }\end{array}$ & 1 & 2,8 & 2,9 & 97,1 \\
\hline & $\begin{array}{c}\text { de } 81 \text { a } 100 \\
\text { euros }\end{array}$ & 1 & 2,8 & 2,9 & 100,0 \\
\hline & Total & 34 & 94,4 & 100,0 & \\
\hline $\begin{array}{l}\text { Perdidos } \\
\text { Sistema }\end{array}$ & & 2 & 5,6 & & \\
\hline Total & & 36 & 100,0 & & \\
\hline
\end{tabular}

15 P-valor $(0,94)$ es mayor que 0,05. Aceptamos la hipótesis nula (Ho) de independencia de variables (no existe asociación entre sexo y gasto mensual en videojuegos). 


\section{Inversión media mujeres videojuegos}

\begin{tabular}{l|c|c|c|c|c} 
& N & Mínimo & MÁximo & media & DEsv. Típ. \\
\hline $\begin{array}{l}\text { ¿Cuál es tu inversión } \\
\text { mensual en videojue- } \\
\text { gos? }\end{array}$ & 34 & 1 & 6 & 2,26 & 1,163 \\
\hline N válido (según lista) & 34 & & & & \\
\hline
\end{tabular}

Fuente: creación propia a partir de datos de encuesta online.FiguRA. 4. Distribución DE FRECUENCIAS INVERSIÓN MEDIA HOMBRES (MENSUAL).

\section{¿Cuál es tu inversión mensual en videojuegos? HOMBRES}

\begin{tabular}{|c|c|c|c|c|c|}
\hline & & FRECUENCIA & PORCENTAJE & $\begin{array}{c}\text { PORCENTAJE } \\
\text { VÁLIDO }\end{array}$ & $\begin{array}{l}\text { PORCENTAJE } \\
\text { ACUMULADO }\end{array}$ \\
\hline & o euros & 197 & 27,2 & 27,2 & 27,2 \\
\hline & $\begin{array}{c}\text { de } 1 \text { a } 20 \\
\text { euros }\end{array}$ & 300 & 41,4 & 41,5 & 68,7 \\
\hline & $\begin{array}{c}\text { de } 21 \text { a } 40 \\
\text { euros }\end{array}$ & 121 & 16,7 & 16,7 & 85,5 \\
\hline & $\begin{array}{c}\text { de } 41 \text { a } 60 \\
\text { euros }\end{array}$ & 51 & 7,0 & 7,1 & 92,5 \\
\hline & $\begin{array}{c}\text { de } 61 \text { a } 80 \\
\text { euros }\end{array}$ & 23 & 3,2 & 3,2 & 95,7 \\
\hline & $\begin{array}{c}\text { de } 81 \text { a } 100 \\
\text { euros }\end{array}$ & 12 & 1,7 & 1,7 & 97,4 \\
\hline & $\begin{array}{c}\text { Más de } 100 \\
\text { euros }\end{array}$ & 19 & 2,6 & 2,6 & 100,0 \\
\hline & Total & 723 & 99,7 & 100,0 & \\
\hline
\end{tabular}




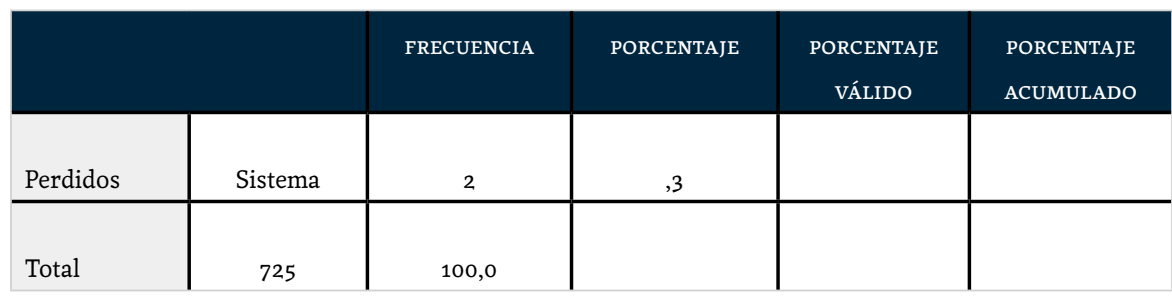

Inversión media hombres videojuegos

\begin{tabular}{l|c|c|c|c|c|} 
& $\mathbf{N}$ & MínIMo & MÁxIMO & MEDIA & DESV. Tí́p. \\
\hline $\begin{array}{l}\text { ¿Cuál es tu inversión } \\
\text { mensual en videojue- } \\
\text { gos? }\end{array}$ & 723 & 1 & 7 & 2,33 & 1,351 \\
\hline N válido (según lista) & 723 & & & & \\
\hline
\end{tabular}

Fuente: creación propia a partir de datos de encuesta online.

Si bien parece existir un cierto consenso acerca de que los comportamientos sexistas están muy presentes en la práctica de juego (TAYLOR, 2006; PEARCE, 2009), este tipo de actitudes no siempre son del todo manifiestas o explícitas. En el diseño y práctica de los videojuegos, como dispositivos culturales, se reproducen y actualizan también los discursos hegemónicos presentes en la sociedad donde se diseñan, practican y consumen. De este modo, los espacios lúdicos son escenarios donde se reinterpretan y reproducen mecanismos de discriminación que rearticulan las prácticas de juego e interacción de jugadores, y especialmente, jugadoras. Son espacios donde las coreografías de género a menudo refuerzan estereotipos y determinados aspectos de los juegos de rol online, como las nociones y representaciones de masculinidad y feminidad.

En la práctica del juego se encuentran desde reprobaciones directas y explícitas hacia las mujeres hasta mecanismos más sutiles de dominación y opresión que se visibilizan en danzas asimétricas, como las actitudes paternalistas en la interacción online del día a día, las jugadoras conviven con diferentes desasosiegos cotidianos que generan algunas problemáticas y malestares, tal y como se muestra en las citas que se ofrecen a continuación.

"A mí algo que me da mucha rabia es que hay comportamientos que dan poco de asco. Por ejemplo, si eres jugadora de rol y no eres muy 
agraciada te tachan de friki o poco femenina, pero si estás buena, para los tíos entonces ya es otra cosa. Pues entonces eres una gamer y eso les mola, eso es guay para ellos. No puede haber algo más machista”. (ENTREVISTA N ${ }^{\mathrm{O}}$ : 7. MUJER, 20 AÑOS, TOLEDO).

"Pues como algo de tonteo o...pues no sé qué...pues la princesa, es que eres nuestra princesa, mi princesa, guerrera...tal. Es como que provoca un morbo especial a los hombres ver a una chica atacando o pegando tiros...” (ENTREVISTA No:9. MUJER, 23 AÑOS, GETAFE).

"Sí claro, y mucho. De hecho pues mira, más sutil o más evidente incluso tus amigos inconscientemente caen en ello. Que si pequeña, que si princesa, que si tengo un regalito para ti...y ahí, baboseándote. $Y$ no, yo ni soy pequeña, ni soy princesa, ni hostias". (ENTREVISTA No: 7 . MUJER, 20 AÑOS, TOLEDO).

"Pero como yo jugaba con mis amigos, sí que eran conscientes de que era yo, $y$ muchas veces pues yo iba un poco más "oh, dios mío, me están matando" y entonces venían todos a salvarme, ¿sabes? Y yo creo que sí, en ese sentido sí...que así un poco era la chica, ¿no? Igual no se me daba tan bien algo y entonces me ayudaban, pero no creo que tampoco haya una gran diferencia, ¿no..?.” (ENTREVISTA No:3. MUJER, 25 AÑOS, MADRID).

\section{EN TERRENO hOSTIL. CONSUMO, REPRESENTACIÓN Y PRÁCtica de VIDEOJUEGOS Y GÉNERO}

Las prácticas sexistas operan de modo diferente cuando el anonimato media la interacción. Pese a que los comportamientos machistas son mucho más explícitos bajo una situación de anonimato ${ }^{16}$, por la impunidad que éste otorga, los resortes más sutiles de la dominación hegemónica también se activan cuando los jugadores se conocen entre sí. Se han hallado dos formas relativamente extendidas de resistencia y discriminación de género, que pueden ser entendidas como conflictos, encontronazos o invasiones de los límites o posicionamientos demarcados en las coreografías de género.

1) En primer lugar no hay que entender el sexismo como la defensa de un espacio. Los escenarios lúdicos de interacción en juegos de rol online 
han sido tradicionalmente espacios de homosocialidad masculina (GOLDING, 2014; KAFAI ET AL., 2008) reservados exclusivamente a los jugadores varones. Son lugares en los que muchos de los hombres que participan no quieren que accedan mujeres para no romper ese espacio masculino de privacidad (rechazo y protección de lo que ellos consideran un espacio de no mujeres). La progresiva inclusión e introducción de usuarias en estos escenarios ha sido respondida con tempestuosas resistencias y percibida por algunos jugadores como una invasión o contaminación del espacio tradicional de juego.

2) Los jugadores que más se identifican con las nociones clásicas de identidad gamer tienden a preservar todavía una visión parcializada que percibe a los videojuegos de rol como una práctica eminentemente masculina. En mayor o menor medida consideran que las jugadoras son menos capaces, hábiles o diestras dentro de este tipo de espacios. Además, según Golding (2014) la progresiva inclusión y diversificación de perfiles de jugadores ha erosionado la identidad tradicional de los gamers (que ocupaba una posición predominante y hegemónica) generando reacciones violentas y de resistencia.

"What we are seeing is the end of gamers, and the viciousness that accompanies the death of an identity. Due to fundamental shifts in the videogame audience, and a move towards progressive attitudes within more traditional areas of videogame culture, the gamer identity has been broken" (GOLDING, 2014: 1).

Las distintas citas mostradas evidencian la existencia de comportamientos sexistas enclavados en la cultura patriarcal más amplia donde el juego toma lugar, en los videojuegos tampoco se dan relaciones igualitarias. Dicha cultura imbricada con los espacios de juego y performatividad, rearticula las lógicas de interacción (más allá del gameplay y las mecánicas de juego) transformando las experiencias y realidades lúdicas. Nuestra apuesta más coreográfica, en línea con Goffman ${ }^{17}$ (1977) y Foster (1998), de entender las relaciones como un baile donde las posiciones de unos condicionan las posiciones de las otras, y donde salirse de los movimientos esperados genera sanciones y presiones. Por ejemplo, cuando las jugadoras se oponen a los mecanismos de dominación existentes, (solicitando que las dejen de llamar "princesa", "reina", "pequeña" o explicitando una situación de acoso) la reacción de los jugadores suele ser muy hostil, mostrando desde rechazo, ira o enfado hasta

17 En "Arrangement Between Sexes" (1977). Por otro lado, en "La presentación de la persona en la vida cotidiana" identifica el sexo como fachada personal. 
conductas más violentas como insultos o actitudes de acoso. De este modo, parece que cuando las jugadoras cuestionan los estereotipos de género los mecanismos más sutiles e implícitos de dominación se manifiestan y se hacen mucho más patentes y visibles. Un ejemplo muy reciente serían toda la serie de ataques, controversias y amenazas ${ }^{18}$ recibidas por activistas feministas, como la bloguera y crítica cultural de videojuegos Anita Sarkeesian. A raíz de lanzar un vídeo ${ }^{19}$ en el que criticaba actitudes misóginas o un tratamiento de los personajes femeninos de los videojuegos como mujeres florero y mera decoración, sufrió una oleada de reacciones hostiles (amenazas y acoso que la forzaron a abandonar su propio domicilio) que pueden ser entendidas bajo esta doble dinámica que entiende el sexismo en los videojuegos principalmente como la protección de un espacio de homosocialidad y una identidad gamer tradicional ${ }^{20}$ que se siente amenazada (aparición de jugadoras y estilos no heteronormativos). Además, este tipo de acosos no se limitan a un caso aislado o concreto, sino que se han ido extendiendo progresivamente a otras mujeres de la industria y el ámbito del videojuego, en lo que se ha conocido popularmente como caso gamergate $e^{21}$ (en la que un conjunto de jugadores y trolls han asediado a diseñadoras, periodistas y mujeres reivindicando unas éticas y entornos de juego más ortodoxos y exclusivos para varones).

De acuerdo a las investigaciones de Taylor (2006), las jugadoras de rol online no encajarían en el estrecho arquetipo de gamer eminentemente masculino, ni en la representación idealizada de los personajes femeninos existentes en los videojuegos, que puede provenir de unas ideas estandarizadas y preconcebidas de feminidad. Según Corneliussen (2008) los estereotipos siguen existiendo en los juegos de rol online, sin embargo, se observa una creciente preocupación por parte de las distintas compañías (cita el ejemplo de Blizzard) para evitar este tipo de prácticas y comportamientos. Las desarrolladoras son progresivamente más conscientes de la gran importancia de las videojugadoras, aunque el proceso está lejos aún de satisfacer las demandas de la comunidad femenina. Numerosas productoras han sido criticadas por no preocuparse por aspectos como la representación de mujeres o la diversidad de perfiles (edades, razas, orientación sexual...). Sin embargo, y

\footnotetext{
18 Disponible en: http://www.polygon.com/2014/8/27/6075679/sarkeesian-driven-out-of-home-byonline-abuse-and-death-threats

19 Vídeo disponible en: https://www.youtube.com/watch?v=5i_RPrgDwMA

20 Identidad gamer notablemente machista.

21 Disponible en: http://deadspin.com/the-future-of-the-culture-wars-is-here-and-itsgamerga-1646145844
} 
afortunadamente, los datos disponibles parecen indicar que los estereotipos están empezando a cambiar poco a poco en el mundo de los videojuegos, mientras la industria empieza a preocuparse por unas representaciones más fidedignas con la compleja y heterogénea variedad de tipos de usuario (especialmente en lo referido a género). Tal y como argumenta Corneliussen:

"The game is clearly moving away from the tradition of hypersexualizing female characters in a male universe, as well as away from one-dimensional presentations of gender within strict gender stereotypes"(2008: 81).

De hecho, la progresiva desdibujación de estereotipos y roles asociados a género se manifiesta claramente si comparamos la evolución de la saga Diablo a lo largo de los distintos lanzamientos. Para empezar, en Diablo III es posible elegir el sexo del avatar, no así en Diablo I o II. Adicionalmente, si se observan las transformaciones en la representación gráfica de los avatares, los personajes femeninos no estarían tan hiper sexualizados ${ }^{22}$ como en las primeras entregas, a la vez que los personajes masculinos comienzan a salir de lo que Corneliussen ha denominado como "masculinidad esteroidal" (steroidal masculinity). Sin embargo, tal y como recuerda Langer (2008), si bien es cierto que determinados estereotipos se han podido diluir parcialmente, los prejuicios asociados a género siguen muy presentes en el ámbito del software lúdico. Concretamente, dicho autor afirma que se manifiestan en dos distintos niveles: en el ámbito de los prejuicios del propio jugador y a nivel de los estereotipos que puedan tener los propios diseñadores y desarrolladores.

"Los diseñadores de videojuegos refuerzan los significados culturales mientras parecen enfrentarse a ellos ${ }^{23}$ "(SCHWARTZ, 2006: 319).

Un ejemplo de cómo los diseñadores pueden reforzar los estereotipos hegemónicos es tratando de trasladar las representaciones y roles asociados a la masculinidad a los personajes femeninos, dotándoles de las cualidades típicamente atribuidas o asociadas a los roles y representaciones de masculinidad. Un caso claro sería el caso de la bárbara en Diablo III (figura 5). Pese

22 Obsérvese la evolución de los avatares en el capítulo VI "Jugando a Diablo", epígrafe número I.I "Personajes y avatares".

23 Traducción propia del original en inglés: "game designers reinforce cultural meanings while appearing to challenge them" (schWARTZ, 2006: 319). 
a que la posibilidad de jugar con una mujer guerrera ha supuesto un avance considerable respecto a títulos anteriores, cabe plantearse la siguiente cuestión: ¿consiste en un personaje que ha superado las barreras de la estereotipia o se ha tratado de adaptar dicha representación a las nociones ideales de masculinidad hipertrofiada? A la vista de ejemplos como el mostrado, parece evidente que aún queda mucho camino por recorrer en lo que a género y videojuegospia o se ha tratado de adaptar dicha representación a las nociones ideales de masculinidad hipertrofiada? A la vista de ejemplos como el mostrado, parece evidente que aún queda mucho camino por recorrer en lo que a género y videojuegos se refiere.

Figura 5. Representación de avatares seleccionables en opción masculina y femenina.
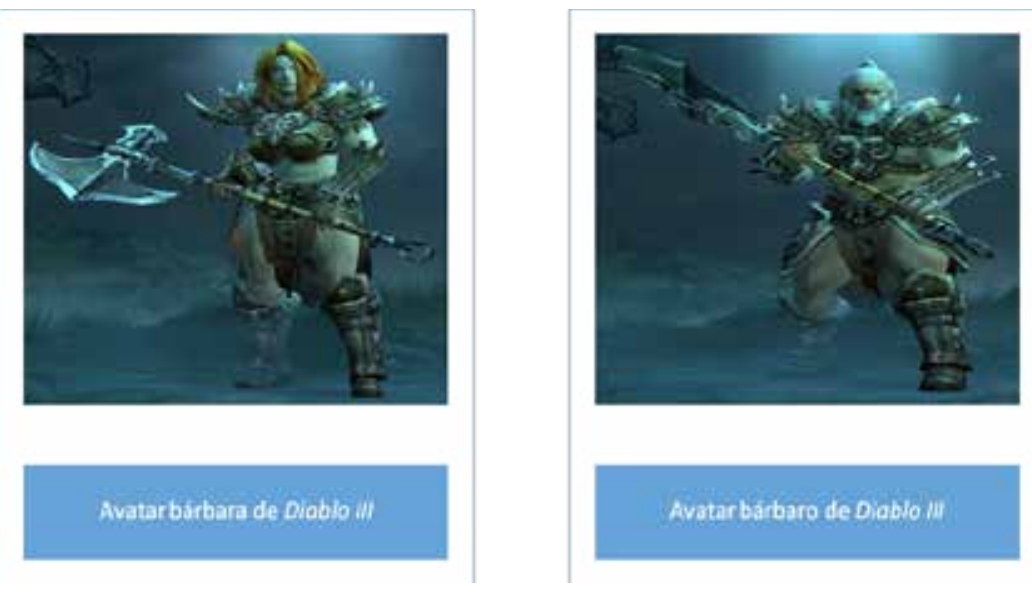

Fuente: propia (pantallazo de Diablo III).

A modo de conclusión del epígrafe parece más que probado que aún queda mucho camino por recorrer en lo que a relaciones de género y videojuegos se refiere. Las contribuciones realizadas en la presente investigación revelan como algunas de las asunciones más asentadas en el campo del género, fans y videojuegos, son parcialmente erróneas o inciertas. En segundo lugar, es muy importante tener en cuenta las recomendaciones que han realizado numerosos autores (CASSEL Y JENKINS, 2000; CORNELIUSSEN, 2008; HJORTH, 2011; EGENFELDT-NIELSEN, SMITH Y TOSCA, 2012) reclamando aproximaciones y modelos de juego más inclusivos, plurales y sensibles a las cuestiones 
de género y diversidad (desprendiéndonos del sistema de prejuicios dominante). Pese a que se han logrado grandes avances en lo referente a acciones, diseños y representaciones más inclusivas e integradoras, es evidente que en el pasado, y aún en la actualidad, la industria ha intentado atraer a las mujeres a los videojuegos reproduciendo estereotipos y roles de género poco acordes con la realidad existente, reforzando unos ideales sesgados de masculinidad y feminidad.

"Luego, me acuerdo que tuve la Game Boy, que la tuvimos los tres hermanos, pero la suya en gris y yo tenía la edición rosa que sacaron, ¿la recuerdas? $Y$ ahí ya jugué verdaderamente por interés [...] Para mis padres era normal que si mis hermanos tenían la Game Boy yo quisiera una también, de esto de que te pilla la fase en que si tus hermanos tienen algo tú también lo quieres...24" (ENTREVISTA MUJER, 20 AÑOS, TOLEDO).

De la misma manera, como mostramos en el caso de la masculinidad esteroidal (CORNELIUSSEN, 2008) o la representación sexista de los personajes femeninos, las jugadoras no siempre se encuentran a gusto con los avatares disponibles, generalmente escasos en versión femenina o sustancialmente hipersexualizados (tal y como se puede observar en la figura 8), lo que si agrada a muchos jugadores heterosexuales que los eligen. Debido a esto, las jugadoras reclaman perfiles de avatares más acordes a la realidad cotidiana y manifiestan la necesidad de incluir representaciones más diversas y complejas. De hecho, tal y como vemos a continuación, existe una clara tendencia entre las usuarias hacia la preferencia en la selección de avatares de sexo femenino, debido principalmente a una cuestión de mayor identificación o representación más acorde a sus expectativas.

"Luego tengo el resto de personajes, todos en versión femenina, porque me gusta aprovechar que te dan la opción de ser mujer en el juego... que muchos juegos no te dan la opción y es como que te sientes un poco... mmmm... no sé si desplazada es la palabra, pero vamos, que me gusta poder elegir ser una mujer." (Entrevista Mujer, 20 años, Toledo).

\footnotetext{
24 Además de la evidencia de las estrategias comerciales para atraer la atención de las mujeres hacia el mundo de los videojuegos, en este extracto nuevamente se vuelve a reflejar como una de las principales vías de acceso de las mujeres a los videojuegos son familiares y amigos.
} 
A la vista de la información recabada a través de la encuesta, los datos recopilados parecen apoyar las hipótesis planteadas anteriormente. En primer lugar, en la figura 6 , se pueden advertir los porcentajes de jugadores totales (hombres y mujeres conjuntamente) que disponen de cada uno de los tipos de avatares seleccionables (hasta un máximo de diez permitido por cuenta de usuario) para ambos modos de juego (clásico e incondicional). En este caso, nos centraremos exclusivamente en el modo de juego normal o clásico por ser, con diferencia, el más frecuente y extendido. Uno de los aspectos más significativos hallados en el caso de Diablo III es que la selección del sexo del avatar se encuentra muy estrechamente vinculada a las habilidades de los personajes. De este modo, las clases asociadas a la fuerza bruta o a las habilidades de combate cuerpo a cuerpo (rudeza, potencia, poca agilidad...), véase bárbaro o monje, tienden a ser seleccionadas bajo un avatar masculino, debido principalmente a que son atributos ligados a las nociones hegemónicas de masculinidad. Por ejemplo, del total de jugadores encuestados, un $69,22 \%$ dispondría de un bárbaro frente a tan sólo un 18,14\% de sujetos que poseería una bárbara (la misma tendencia se manifiesta en el caso del monje/ monja). Por el contrario, los personajes asociados a habilidades de combate a distancia (inteligencia, estrategia, agilidad, poca resistencia física), tales como el mago o el cazador de demonios, tienden a ser seleccionados en sexo femenino. Podemos concluir, por tanto, que los estereotipos e imaginarios dominantes de masculinidad y feminidad condicionan no sólo la apariencia o representación de los avatares, sino que implican una adopción de roles o actitudes esperadas que se visibilizan a través de las habilidades, capacidades y actuaciones (GOFFMAN, 1959). Adicionalmente, se evidencia nuevamente que las jugadoras muestran su preferencia por un estilo de juego más estratégico o de habilidades de combate a distancia donde las capacidades intelectuales priman sobre la fuerza bruta. 
Figura 6. Porcentajes de jugadores que disponen de cada tipo de avatar por clase y sexo ${ }^{25}$ (respondido: 784, omitido: 165).

\begin{tabular}{l|c|c}
\hline & MODO CLÁssico & MODO INCONDICIONAL \\
\hline Bárbara & $18,14 \% 142$ & $18,84 \% 68$ \\
\hline Bárbaro & $69,22 \% 542$ & $29,09 \% 105$ \\
\hline Cazadora de demon & $43,81 \% 343$ & $16,90 \% 61$ \\
\hline Cazador de demon & $36,65 \% 287$ & $12,74 \% 46$ \\
\hline Maga & $43,04 \% 337$ & $18,28 \% 66$ \\
\hline Mago & $37,55 \% 294$ & $15,51 \% 56$ \\
\hline Médica bruja & $17,50 \% 137$ & $18,28 \% 66$ \\
\hline Médico brujo & $52,23 \% 409$ & $18,01 \% 65$ \\
\hline Monje (femen) & $29,50 \% 231$ & $18,84 \% 68$ \\
\hline Monge (mascu) & $47,13 \% 369$ & $18,56 \% 67$ \\
\hline
\end{tabular}

Fuente: elaboración propia a par tir de encuesta online.

Con el propósito de enriquecer y sustentar los hallazgos obtenidos respecto a la preferencia de las jugadoras de Diablo III por avatares femeninos, se ha optado por segmentar la muestra seleccionando únicamente los casos de mujeres. A continuación, se muestra qué porcentajes de jugadoras disponen de cada tipo de avatar ${ }^{26}$.

25 Los porcentajes relativos de jugadores que disponen de cada tipo de avatar por clase (bárbaro, cazador de demonios, mago, médico brujo y monje) y sexo (masculino o femenino) aparecen en negrita, mientras que el número total en bruto de jugadores que poseen cada tipo de avatar aparecería en gris claro. Por ejemplo: el 18,14\% de jugadores de Diablo III disponen de un avatar de bárbara, lo que supondría 142 jugadores de la muestra total de respuestas obtenidas para dicha pregunta (784 respondientes).

26 Se recuerda que cada jugador o jugadora puede tener un máximo de 10 avatares entre las clases, sexos y modos de juego existentes en Diablo III. 
Figura 7. Porcentajes de jugadoras que disponen de cada tipo de avatar por clase y sexo $(n=36)$.

\begin{tabular}{c|c|c}
\hline & CLASE & $\%$ \\
\cline { 2 - 3 } & Bárbara & $38,9 \%$ \\
\cline { 2 - 3 } & Bárbaro & $33,3 \%$ \\
\cline { 2 - 3 } & Cazadora & $52,8 \%$ \\
\cline { 2 - 3 } AVATARES \\
SELECIONADOS \\
\cline { 2 - 3 } POR JUGADORAS \\
\cline { 2 - 3 } (MODO CLÁSSICO)
\end{tabular}

Fuente: elaboración propia a partir de encuesta online.

Tal y como se refleja en la figura 7, la elección de personajes de sexo femenino es significativamente superior entre las jugadoras, especialmente en el caso de la maga $(52,8 \%)$ frente al mago (8,3\%). Pero ¿qué ocurre si se observa el caso de la bárbara? A la vista de los datos, los porcentajes arrojados se encuentran mucho más parejos que en el primer ejemplo ofrecido (38,9\% frente al $33,3 \%)$. Una de las explicaciones fundamentales, radicaría nuevamente en las hipótesis apuntadas previamente, y es que las nociones de feminidad asociadas a cada perfil de avatar provocarían que las mujeres se identifiquen mucho más con las capacidades, estilo de juego o apariencia de la maga (figura 8, primer personaje por la izquierda) frente a una ruda guerrera (véase figura 5). 
Figura 8. Personajes seleccionables. Maga, médico brujo, cazadora de demonios, guerrero y monje.

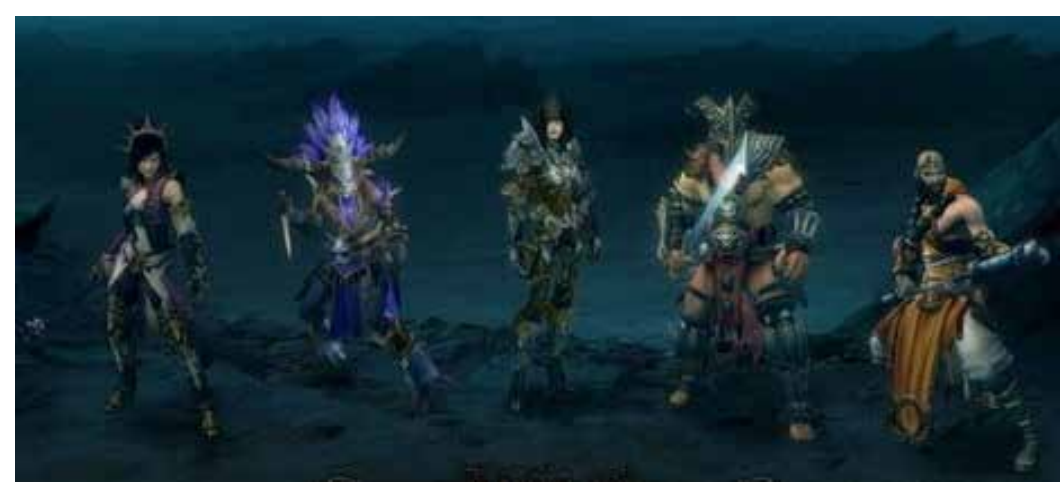

Fuente: elaboración propia

Por el contrario, entre usuarios de sexo masculino dicha tendencia no se encuentra tan acentuada, ya que los jugadores varones son mucho más proclives a seleccionar avatares femeninos, de hecho, el porcentaje de magas o cazadoras de demonios jugadas por hombres es sustancialmente superior al de su versión masculina. La observación etnográfica, junto a la exploración a través de entrevistas en profundidad, revelaron la existencia de numerosos casos en los que jugadores (hombres) se sienten atraídos por los diseños sexualizados de los avatares femeninos, incentivando su selección como personaje-avatar, tendencia mucho menos frecuente en el caso de las mujeres, que no suelen seleccionar sus avatares por razones de atracción física.

\section{CONCLUSIONES}

A lo largo del trabajo hemos entendido las prácticas de juego online como una suerte de coreografía colectiva (agencia compartida) entre las jugadoras y jugadores, los fans, los dispositivos tecnológicos, las plataformas y las condiciones específicas de diseño y comercialización; un danza multitudinaria donde se manifiestan y operan variedad de conflictos, potencialidades, resistencias y controversias. Espacios y relaciones recíprocas donde se construye género y donde se cuestionan atribuciones y expectativas dominantes a través de prácticas lúdicas y posicionamientos coreográficos.

Desde un acercamiento al género que nos devuelve a las relaciones cotidianas, hemos tratado de evidenciar la existencia de prejuicios, constreñi- 
mientos y comportamientos sexistas existentes en otras esferas de la vida cotidiana y relaciones de género (un espacio donde el jugador varón es quién tiende a marcar los ritmos y pasos frente a las jugadoras). El sexismo está aún muy extendido en el servidor español de Diablo y concretamente se han encontrado dos formas comunes de resistencia y limitación de género que pueden ser abordadas desde la perspectiva coreográfica. En primer lugar se evidencias conflictos y "pisotones" ante la defensa de un espacio de homosocialidad (reservado en exclusiva a los hombres) y una identidad gamer de raíces y bases machistas (donde la progresiva inclusión de perfiles más heterogéneos ha erosionado la identidad clásica que ocupaba una posición predominante y hegemónica en el escenario). Cuando las jugadoras se oponen a los mecanismos de dominación existentes encarnados en actitudes y comentarios que remiten a los posicionamientos del varón protector y poderoso, y de la mujer como "damisela en apuros", la reacción de los jugadores es muy hostil. De este modo, parece que cuando las mujeres cuestionan las estructuras de poder hegemónicas, los mecanismos más sutiles e implícitos de dominación se manifiestan y se hacen mucho más patentes y visibles.

Parece evidente que aún queda mucho camino por recorrer en lo que a género y videojuegos se refiere. Las contribuciones realizadas en este trabajo revelan como algunas de las asunciones más asentadas en el campo son cuestionables o inciertas. Pese a que se han logrado grandes avances en lo referente a diseños y representaciones más inclusivas (introducción de avatares femeninos), la industria ha intentado atraer a las mujeres reproduciendo estereotipos y roles de género poco acordes con la realidad existente, reforzando unos ideales sesgados de feminidad y obviando los desequilibrios y conflictos de género existentes, tal y como hemos tratado de reflejar a través de nuestra apuesta coreográfica.

\section{REFERENCIAS}

AARSETH, ESPEN (2008). A Hollow World: World of Warcraft as spatial practice en CORNELIUSSEN, Hilde, RETTBERG, Jill (Ed.), Digital culture, play, and identity: A world of warcraft reader, Cambridge: MIT Press, pp.111-122.

BECKER, HOWARD (1995). El poder de la inercia. Apuntes de Investigación del CECYP, 15, pp. 99-111.

BOLTER, JAY, GRUSIN, RICHARD (2000). Remediation. Understanding New Media. Cambridge: MIT Press.

BUTLER, JUDITH (1990). Gender Trouble: Feminism and the Subversion of 
Identity. New York: Routledge.

CASSEL, JUSTINE, JENKINS, HENRY (2000). From Barbie to Mortal Kombat: Gender and Computer Games. Cambridge: The MIT Press.

CORNELIUSSEN, HILL, RETTBERG, JILDE (2008). Digital culture, play, and identity: A world of warcraft reader, Cambridge: MIT Press.

DENZIN, NORMAN (1970). Sociological Methods: a Source Book. Chicago: Aldine Publishing Company.

EGENFELDT-NIELSEN, SIMON, SMITH, JONAS, TOSCA, SUSANA (2012). Understanding videogames: The essential introduction. Nueva York: Routledge (segunda edición).

ERMI, LAURA, MÄYRÄ, FRANS (2005). Fundamental Components of the Gameplay Experience: Analysing Immersion. Tampere: Universidad de Tampere. Dirección: http://www.uta.fi/ tlilma/gameplay_experience.pdf (Última consulta: 20 de noviembre 2014)

FOSTER, SUSAN LEIGH (1998). Choreographies of Gender. Signs, 24(1), pp 1-33. FOUCAULT, MICHEL (1983). The Subject and Power: Beyond Structuralism and Hermeneutics, en DREYFUS Hubert, RABINOW, Paul (Ed.)., Beyond Structuralism and Hermeneutics, Chicago: The University of Chicago Press, pp.208-226.

GOLDING, DAN (2014). The End of Gamers. Dirección: http://dangolding. tumblr.com/post/95985875943/the-end-of-gamers (Última consulta: 20 de noviembre de 2014)

GOFFMAN, ERVING (1977). The Arrangement between the Sexes. Theory and Society, 4 (3), pp.301-331.

GOFFMAN, ERVING. (1959) (1971). La presentación de la persona en la vida cotidiana, Buenos Aires: Amorrortu.

KAFAI, YASMIN., HEETER, CARRIE., DENNER, JILL., SUN, JEN. (Ed.)., Beyond Barbie and Mortal Kombat: New perspectives on gender and gaming, Cambridge: MIT Press, pp. 160-177.

KENNEDY, HELEN (2002). Lara Croft: Feminist Icon or Cyberbimbo? On the Limits of Textual Analysis. Game Studies, 2 (2). Dirección: http://www. gamestudies.org/0202/kennedy/ (Última consulta: 19 de noviembre 2014) LANGER, JESSICA (2008). The familiar and the foreign: Playing (post) colonialism in world of Warcraft, en CORNELIUSSEN, Hilde, RETTBERG, Jill (Ed.), Digital culture, play, and identity: A world of warcraft reader. Cambridge: TheMIT Press, pp. 87-108.

LASÉN, AMPARO (2012). Autofotos. Subjetividades y Medios Sociales, en García-Canclini, Nestor, Cruces, Francisco y Guadarrama, Maritza (Ed.), Jóvenes, culturas urbanas y redes digitales. Prácticas emergentes en las 
artes, el campo editorial y la música. Madrid: Ariel, pp.243-262.

HEETER, CARRIE. (2008). Changing Girls, Changing Games, en KAFAI, Yasmin., HEETER, Carrie., DENNER, Jill., SUN, Jen. (Ed.)., Beyond Barbie and Mortal Kombat: New perspectives on gender and gaming, Cambridge: MIT Press, 160-177. (nombres propios)

LATOUR, BRUNO (1992). Where Are the Missing Masses? The Sociology of a Few Mundane Artifacts en Wiebe E. BIJKER y John LAW, (Ed.), Shaping Technology/Building Society: Studies in Sociotechnical Change, Cambridge, Mass.: MIT Press, pp. 225-258.

LAUREL, BRENDA (2006). Why didn't girls play videogames? Brenda Laurel on TED.com, Rescatado de http://blog.ted.com/2009/03/why_didnt_girls. php

MÄYRÄ, FRANS. (2009). La experiencia de juego en contexto. Sobre los contextos socioculturales del significado en el juego digital, en Aranda, D., SánchezNavarro, J. (Ed.)., Aprovecha el tiempo y juega: Algunas claves para entender los videojuegos. Barcelona: UOCpress, pp.129-144.

PEARCE, CELIA. (2009). Communities of play: Emergent cultures in multiplayer games and virtual worlds. Massachusetts: MIT Press.

PUENTE, HÉCTOR., FERNÁNDEZ, MARTA. (2013). User Generated Content: A Situated Production of Video Walkthroughs on Youtube, en MITGUTSCH, Konstantin., HUBER, Simon., WIMMER, Jeffrey., WAGNER, Michael., ROSENSTINGL, Hubert. (Ed.), Context Matters!Exploring and Reframing Games in Context. Proceedings of the 7th Vienna Games Conference 2013, FROG 2013 Viena: New Academic Press, pp.136-147.

SARKEESIAN, ANITA. (2013). Damsel in Distress Rescatado de http://www. feministfrequency.com/2013/03/damsel-in-distress-part-1/

SCHOTT, GARETH. HORREL, KIRSTY. (2000). Girl Gamers and their Relathionship with the Gaming Culture. Convergence, 6, (4), 36-54.

SCHWARTZ, LEIGH. (2006). Fantasy, Realism, and the Other in Recent Video Games. Space and Culture (SAGE), 9, pp.313-321.

TAYLOR, T.L. (2006). Play Between Worlds: Exploring Online Game Culture, Cambridge: MIT Press.

TURKLE, SHERRY. (1995). Life on the Screen: Identity in the Age of the Internet, Londres: Phoenix. 